\title{
The relative contribution of cerebrospinal fluid malabsorption and obstruction in the development of hydrocephalus in human neonates with spina bifida
}

\author{
DA Sival*1, EW Hoving'2, B Stoffel-Wagner ${ }^{3}$, AF Bos ${ }^{4}$, A Teelken ${ }^{5}$, \\ OF Brouwer ${ }^{1}$, P Bartmann ${ }^{6}$ and A Heep ${ }^{6}$
}

\begin{abstract}
Address: ${ }^{1}$ Pediatric Neurology, University Medical Center Groningen, Netherlands, ${ }^{2}$ Neurosurgery, University Medical Center Groningen, Netherlands, ${ }^{3}$ Biochemistry, University Hospital Bonn, Germany, ${ }^{4}$ Neonatology, University Medical Center Groningen, Netherlands, ${ }^{5}$ Neurochemistry University Medical Center Groningen, Netherlands and ${ }^{6}$ Neonatology, University Hospital Bonn, Germany

Email: DA Sival* - dasival@hotmail.com

* Corresponding author
\end{abstract}

from 49th Annual Meeting of the Society for Research into Hydrocephalus and Spina Bifida Barcelona, Spain, 29 June - 2 July 2005

Published: 30 December 2005

Cerebrospinal Fluid Research 2005, 2(SuppI I):S34 doi:I0.1 186/1743-8454-2-SI-S34

\section{Background}

The pathogenesis of hydrocephalus in human neonates with spina bifida (SB) is incompletely understood. In HT$\mathrm{x}$ rats, a model for congenital hydrocephalus, initial cerebrospinal fluid (CSF) malabsorption and CSF obstruction seemingly interplay in the pathogenesis. In human hydrocephalic SB neonates, assessment of CSF biomarkers may help to distinct between the relative contribution of CSF malabsorption and obstruction in the development of hydrocephalus. In this respect, concentrations of aminoterminal propeptide of type 1 collagen (PINP), a biomarker for arachnoideal fibrosis, has been shown to relate to CSF malabsorption in human hydrocephalic neonates. Additionally, high concentrations of transforming growth factor $\beta 1$ (TGF $\beta 1$, involved in cellular proliferation and migration) are associated with CSF malabsorption (mice), whereas low TGF $\beta 1$ concentrations are associated with congenital CSF obstruction (HT-x rats). In human hydrocephalic SB neonates, it is unclear how PINP and TGF $\beta 1$ relate to the development of hydrocephalus. Elucidation of the pathogenesis of hydrocephalus in SB neonates may be important for the timing and optimalization of therapeutical strategies.

\section{Objective}

To determine the relative contribution of CSF malabsorption and obstruction during the development of hydrocephalus in SB neonates

\section{Materials and methods}

CSF concentrations of PINP (radio isotope assay) and TGF $\beta 1$ (ELISA) were assessed in hydrocephalic SB neonates $(\mathrm{n}=10)$, and compared with PINP and TGF $\beta 1$ concentrations in hydrocephalic neonates with aqueduct stenosis (CSF obstruction $(\mathrm{n}=4)$ ), and with fetal intraventricular haemorrhages (CSF malabsorption $(n=4)$ ). Interleukin-6 concentrations (IL-6; ELISA) in CSF were determined in all samples to estimate the proinflammatory state.

\section{Results}

In CSF from SB-hydrocephalus patients, concentrations of PINP and TGF $\beta 1$ were $~ 80 \%$ and $~ 50 \%$ lower than in malabsorption hydrocephalus (median values: PINP 181 ng/ml vs. $1074 \mathrm{ng} / \mathrm{ml}$, resp., $\mathrm{p}=0.002$; TGF $\beta 1104$ vs. 277 $\mathrm{pg} / \mathrm{ml}$, resp., $\mathrm{p}=0.03$ ). CSF PINP and TGF $\beta 1$ concentrations did not significantly differ between SB-hydrocephalus and obstruction hydrocephalus. Median IL-6 CSF concentrations did not differ between the groups.

\section{Conclusion}

Present data on CSF biomarkers strongly indicate that CSF obstruction contributes more to the development of hydrocephalus in SB neonates than arachnoideal CSF malabsorption. These data in hydrocephalic SB neonates are compatible with the concept that low CSF growth factor concentrations and CSF obstruction to the cortex may relate to cortical impairment 